This item was submitted to Loughborough's Research Repository by the author.

Items in Figshare are protected by copyright, with all rights reserved, unless otherwise indicated.

\title{
k-spectra of weakly-c-balanced words
}

PLEASE CITE THE PUBLISHED VERSION

https://doi.org/10.1007/978-3-030-24886-4_20

PUBLISHER

Springer

VERSION

AM (Accepted Manuscript)

PUBLISHER STATEMENT

The final authenticated version is available online at https://doi.org/10.1007/978-3-030-24886-4_20.

LICENCE

CC BY-NC-ND 4.0

\section{REPOSITORY RECORD}

Day, Joel, Pamela Fleischmann, Florin Manea, and Dirk Nowotka. 2019. "K-spectra of Weakly-c-balanced Words". Loughborough University. https://hdl.handle.net/2134/15028887.v1. 


\title{
$k$-Spectra of Weakly-c-Balanced Words
}

\author{
Joel D. Day ${ }^{1}$, Pamela Fleischmann ${ }^{2}$, Florin Manea ${ }^{2}$, and Dirk Nowotka ${ }^{2}$ \\ 1 Loughborough University, UK, J.Day@lboro.ac.uk \\ 2 Kiel University, Germany $\{\mathrm{fpa}, \mathrm{flm}, \mathrm{dn}\}$ @informatik. uni-kiel.de
}

\begin{abstract}
A word $u$ is a scattered factor of $w$ if $u$ can be obtained from $w$ by deleting some of its letters. That is, there exist the (potentially empty) words $u_{1}, u_{2}, \ldots, u_{n}$, and $v_{0}, v_{1}, . ., v_{n}$ such that $u=u_{1} u_{2} \ldots u_{n}$ and $w=v_{0} u_{1} v_{1} u_{2} v_{2} \ldots u_{n} v_{n}$. We consider the set of length- $k$ scattered factors of a given word $w$, called here $k$-spectrum and denoted $\operatorname{ScatFact}_{k}(w)$. We prove a series of properties of the sets $\operatorname{ScatFact}_{k}(w)$ for binary weakly-0balanced and, respectively, weakly-c-balanced words $w$, i.e., words over a two-letter alphabet where the number of occurrences of each letter is the same, or, respectively, one letter has c-more occurrences than the other. In particular, we consider the question which cardinalities $n=\left|\operatorname{ScatFact}_{k}(w)\right|$ are obtainable, for a positive integer $k$, when $w$ is either a weakly-0-balanced binary word of length $2 k$, or a weakly-cbalanced binary word of length $2 k-c$. We also consider the problem of reconstructing words from their $k$-spectra.
\end{abstract}

\section{Introduction}

Given a word $w$, a scattered factor (also called scattered subword, or simply subword in the literature) is a word obtained by removing one or more factors from $w$. More formally, $u$ is a scattered factor of $w$ if there exist $u_{1}, \ldots, u_{n} \in$ $\Sigma^{*}, v_{0}, \ldots, v_{n} \in \Sigma^{*}$ such that $u=u_{1} u_{2} \ldots u_{n}$ and $w=v_{0} u_{1} v_{1} u_{1} \ldots u_{n} v_{n}$. Consequently a scattered factor of $w$ can be thought of as a representation of $w$ in which some parts are missing. As such, there is considerable interest in the relationship of a word and its scattered factors from both a theoretical and practical point of view. For an introduction to the study of scattered factors, see Chapter 6 of [9]. On the one hand, it is easy to imagine how, in any situation where discrete, linear data is read from an imperfect input - such as when sequencing DNA or during the transmission of a digital signal - scattered factors form a natural model, as multiple parts of the input may be missed, but the rest will remain unaffected and in-sequence. For instance, various applications and connections of this model in verification are discussed in [14,6] within a language theoretic framework, while applications of the model in DNA sequencing are discussed in [4] in an algorithmic framework. On the other hand, from a more algebraic perspective, there have been efforts to bridge the gap between the non-commutative field of combinatorics on words with traditional commutative mathematics via Parikh matrices (cf. e.g., $[11,13]$ ) which are closely related to, and influenced by the topic of scattered factors. 
The set (or also in some cases, multi-set) of scattered factors of a word $w$, denoted $\operatorname{ScatFact}(w)$ is typically exponentially large in the length of $w$, and contains a lot of redundant information in the sense that, for $k^{\prime}<k \leq|w|$, a word of length $k^{\prime}$ is a scattered factor of $w$ if and only if it is a scattered factor of a scattered factor of $w$ of length $k$. This has led to the idea of $k$-spectra: the set of all length- $k$ scattered factors of a word. For example, the 3 -spectrum of the word $a b a b b b$ is the set $\{a a b, a b a, a b b, b a b, b b b\}$. Note that unlike some literature, we do not consider the $k$-spectra to be the multi-set of scattered factors in the present work, but rather ignore the multiplicities. This distinction is non-trivial as there are significant variations on the properties based on these different definitions (cf. e.g., [10]). Also, the notion of $k$-spectra is closely related to the classical notion of factor complexity of words, which counts, for each positive integer $k$, the number of distinct factors of length $k$ of a word. Here, the cardinality of the $k$-spectrum of a word gives the number of the word's distinct scattered factors of length $k$.

One of the most fundamental questions about $k$-spectra of words, and indeed sets of scattered factors in general, is that of recognition: given a set $S$ of words (of length $k$ ), is $S$ the subset of a $k$-spectrum of some word? In general, it remains a long standing goal of the theory to give a "nice" descriptive characterisation of scattered factor sets (and $k$-spectra), and to better understand their structure [9]. Another fundamental question concerning $k$-spectra, and one well motivated in several applications, is the question of reconstruction: given a word $w$ of length $n$, what is the smallest value $k$ such that the $k$-spectrum of $w$ is uniquely determined? This question was addressed and solved successively in a variety of cases. In particular, in [3], the exact bound of $\frac{n}{2}+1$ is given in the general case. Other variations, including for the definition of $k$-spectra where multiplicities are also taken into account, are considered in [10], while [7] considers the question of reconstructing words from their palindromic scattered factors.

In the current work, we consider $k$-spectra in the restricted setting of a binary alphabet $\Sigma=\{\mathrm{a}, \mathrm{b}\}$. For such an alphabet, we can always identify the natural number $c \in \mathbb{N}_{0}$ which describes how weakly balanced a word is: $c$ is the difference between the amount of as and bs. Thus, it seems natural to categorise all words over $\Sigma$ according to this difference: a binary word where one letter has exactly $c$ more occurrences than the other one is called weakly-c-balanced. In Section 3 the cardinalities of $k$-spectra of weakly-c-balanced words of length $2 k-c$ are investigated. Our first results concern the minimal and maximal cardinality ScatFact ${ }_{k}$ might have. We show that the cardinality ranges for weakly-0-balanced between $k+1$ and $2^{k}$, and determine exactly for which words of length $2 k$ these values are reached. In the case of weakly-c-balanced words, we are able to replicate the result regarding the minimal cardinality of ScatFact ${ }_{k}$, but the case of maximal cardinality seems to be more complicated. To this end, it seems that the words containing many alternations between the two letters of the alphabet have larger sets ScatFact $k$. Therefore, we first investigate the scattered factors of the words which are prefixes of $(a b)^{\omega}$ and give a precise description of all scattered factors of any length of such words. That is, not only we compute the cardinality of 
$\operatorname{ScatFact}_{k}(w)$, for all such words $w$, but also describe a way to obtain directly the respective scattered factors, without repetitions. We use this to describe exactly the sets ScatFact $i$ for the word $(\mathrm{ab})^{k-c} \mathrm{a}^{c}$, which seems a good candidate for a weakly-c-balanced word with many distinct scattered factors.

Further, in Section 4, we explore more the cardinalities of $\operatorname{ScatFact}_{k}(w)$ for weakly-0-balanced words $w$ of length $2 k$. We obtain for these words that the smallest three numbers which are possible cardinalities for their $k$-spectra are $k+1,2 k$, and $3 k-3$, thus identifying two gaps in the set of such cardinalities. Among other results on this topic, we show that for every constant $i$ there exist a word $w$ of length $2 k$ such that $\left|\operatorname{ScatFact}_{k}(w)\right| \in \Theta\left(n^{i}\right)$; we also show how such a word can be constructed.

Finally, in Section 5, we also approach the question of reconstructing weakly0 -balanced words from $k$-spectra in the specific case that the spectra are also limited to weakly-0-balanced words only. While we are not able to resolve the question completely, we conjecture that the situation is similar to the general case: the smallest value $k$ such that the $k$-spectrum of $w$ is uniquely determined is $k=\frac{|w|}{2}+1$ if $\frac{|w|}{2}$ is odd and $k=\frac{|w|}{2}+2$, otherwise, in the case when $w$ contains at most two blocks of bs.

After introducing a series of basic definitions, preliminaries, and notations, the organisation of the paper follows the description above. The proofs can be found in [2].

\section{Preliminaries}

Let $\mathbb{N}$ be the set of natural numbers, $\mathbb{N}_{0}=\mathbb{N} \cup\{0\}$, and let $\mathbb{N}_{>k}$ be all natural numbers greater than or equal to $k$. Let $[n]$ denote the set $\{1, \ldots, n\}$ and $[n]_{0}=$ $[n] \cup\{0\}$ for an $n \in \mathbb{N}$.

We consider words $w$ over the alphabet $\Sigma=\{\mathrm{a}, \mathrm{b}\} . \Sigma^{*}$ denotes the set of all finite words over $\Sigma$, also called binary words. $\Sigma^{\omega}$ the set of all infinite words over $\Sigma$, also called binary infinite words. The empty word is denoted by $\varepsilon$ and $\Sigma^{+}$is the free semigroup $\Sigma^{*} \backslash\{\varepsilon\}$. The length of a word $w$ is denoted by $|w|$. Let $\Sigma^{\leq k}:=\left\{w \in \Sigma^{*}|| w \mid \leq k\right\}$ and $\Sigma^{k}$ be the set of all words of length exactly $k \in \mathbb{N}$. The number of occurrences of a letter a $\in \Sigma$ in a word $w \in \Sigma^{*}$ is denoted by $|w|_{\mathrm{a}}$. The $\mathrm{i}^{\text {th }}$ letter of a word $w$ is given by $w[i]$ for $i \in[|w|]$. For a given word $w \in \Sigma^{n}$ the reversal of $w$ is defined by $w^{R}=w[n] w[n-1] \ldots w[2] w[1]$. The powers of $w \in \Sigma^{*}$ are defined recursively by $w^{0}=\varepsilon, w^{n}=w w^{n-1}$ for $n \in \mathbb{N}$.

A word $w \in \Sigma^{*}$ is called weakly-c-balanced if $\left.|| w\right|_{a}-|w|_{b} \mid=c$ for $c \in \mathbb{N}_{0}$. Thus weakly-0-balanced words have the same number of as and bs. Let $\Sigma_{w z b}^{*}$ be the set of all weakly-0-balanced words over $\Sigma$. For example, abaa is weakly-2-balanced, aba is weakly-1-balanced, while abbaba is weakly-0-balanced.

A word $u \in \Sigma^{*}$ is a factor of $w \in \Sigma^{*}$, if $w=x u y$ holds for some words $x, y \in \Sigma^{*}$. Moreover, $u$ is a prefix of $w$ if $x=\varepsilon$ holds and a suffix if $y=\varepsilon$ holds. The factor of $w$ from the $\mathrm{i}^{\text {th }}$ to the $\mathrm{j}^{\text {th }}$ letter will be denoted by $w[i . . j]$ for $0 \leq i \leq j \leq|w|$. Given a letter a $\in \Sigma$ and a word $w \in \Sigma^{*}$, a block of a is a factor $u=w[i . . j]$ with $u=\mathrm{a}^{j-i}$, such that either $i=1$ or $w[i-1]=\mathrm{b} \neq \mathrm{a}$ and either 
$j=|w|$ or $w[j+1]=\mathrm{b} \neq \mathrm{a}$. For example the word abaaabaabb has 3 a-blocks and 3 b-blocks. Scattered factors and $k$-spectra are defined as follows.

Definition 1. A word $u=a_{1} \ldots a_{n} \in \Sigma^{n}$, for $n \in \mathbb{N}$, is a scattered factor of $a$ word $w \in \Sigma^{+}$if there exists $v_{0}, \ldots, v_{n} \in \Sigma^{*}$ with $w=v_{0} a_{1} v_{1} \ldots v_{n-1} a_{n} v_{n}$. Let $\operatorname{ScatFact}(w)$ denote the set of $w$ 's scattered factors and consider additionally $\operatorname{ScatFact}_{k}(w)$ and $\operatorname{ScatFact}_{\leq k}(w)$ as the two subsets of $\operatorname{ScatFact}(w)$ which contain only the scattered factors of length $k \in \mathbb{N}$ or the ones up to length $k \in \mathbb{N}$.

The sets $\operatorname{ScatFact}_{\leq k}(w)$ and $\operatorname{ScatFact}_{k}(w)$ are also known as full $k$-spectrum and, respectively, $k$-spectrum of a word $w \in \Sigma^{*}$ (see [1], [10], [12]) and moreover, scattered factors are often called subwords or scattered subwords. Obviously the $k$-spectrum is empty for $k>|w|$ and contains exactly $w$ 's letters for $k=1$ and only $w$ for $k=|w|$. Considering the word $w=$ abba, the other spectra are given by $\operatorname{ScatFact}_{2}(w)=\left\{\mathrm{a}^{2}, \mathrm{~b}^{2}, \mathrm{ab}, \mathrm{ba}\right\}$ and $\operatorname{ScatFact}_{3}(w)=\left\{\mathrm{ab}^{2}, \mathrm{aba}, \mathrm{b}^{2} \mathrm{a}\right\}$.

It is worth noting that if $u$ is a scattered factor of $w$, and $v$ is a scattered factor of $u$, then $v$ is a scattered factor of $w$. Additionally, notice two important symmetries regarding $k$-spectra. For $w \in \Sigma^{*}$ and the renaming morphism ${ }^{-}$: $\Sigma \rightarrow \Sigma$ with $\overline{\mathrm{a}}=\mathrm{b}$ and $\overline{\mathrm{b}}=\mathrm{a}$ we have $\operatorname{ScatFact}\left(w^{R}\right)=\left\{u^{R} \mid u \in \operatorname{ScatFact}(w)\right\}$ and $\operatorname{ScatFact}(\bar{w})=\{\bar{u} \mid u \in \operatorname{ScatFact}(w)\}$. Thus, from a structural point of view, it is sufficient to consider only one representative from the equivalence classes induced by the equivalence relation where $w_{1}$ is equivalent to $w_{2}$ whenever $w_{2}$ is obtained by a composition of reversals and renamings from $w_{1}$. Considering w.l.o.g. the order a $<\mathrm{b}$ on $\Sigma$, we choose the lexicographically smallest word as representative from each class. As such, we will mostly analyse the $k$-spectra of words starting with a. We shall make use of this fact extensively in Section 4 .

\section{Cardinalities of $k$-Spectra of Weakly-c-Balanced Words}

In the current section, we consider the combinatorial properties of $k$-spectra of weakly-c-balanced finite words. In particular, we are interested in the cardinalities of the $k$-spectra and in the question: which cardinalities are (not) possible? Since the $k$-spectra of $\mathrm{a}^{n}$ and $\mathrm{b}^{n}$ are just $\mathrm{a}^{k}$ and $\mathrm{b}^{k}$ respectively for all $n \in \mathbb{N}_{0}$ and $k \in[n]_{0}$, we assume $|w|_{\mathrm{a}},|w|_{\mathrm{b}}>0$ for $w \in \Sigma^{*}$. It is a straightforward observation that not every subset of $\Sigma^{k}$ is a $k$-spectrum of some word $w$. For example, for $k=2$, aa and bb can only be scattered factors of a word containing both as and bs, and therefore having either ab or ba as a scattered factor as well. Thus, there is no word $w$ such that $\operatorname{ScatFact}_{2}(w)=\{\mathrm{aa}, \mathrm{bb}\}$.

In general, for any word containing only as or only bs, there will be exactly one scattered factor of each length, while for words containing both a's and b's, the smallest $k$-spectra are realised for words of the form $w=\mathrm{a}^{n} \mathrm{~b}$ (up to renaming and reversal), for which $\operatorname{ScatFact}_{k}(w)=\left\{\mathrm{a}^{k}, \mathrm{a}^{k-1} \mathbf{b}\right\}$ for each $k \in[|w|]$. On the other hand, as Proposition 5 shows, the maximal $k$-spectra are those containing all words of length $k$ - and hence have size $2^{k}$, achieved by e.g. $w=(\mathrm{ab})^{n}$ for $n \geq k$. Note that when weakly-0-balanced words are considered, the same 
maximum applies, since $(\mathrm{ab})^{n}$ is weakly-0-balanced, while the minimum does not, since $\mathrm{a}^{n} \mathrm{~b}$ is not weakly-0-balanced.

It is straightforward to enumerate all possible $k$-spectra, and describe the words realising them for $k \leq 2$, hence we shall generally consider only $k$-spectra in the sequel for which $k \geq 3$. Our first result generalises the previous observation about minimal-size $k$-spectra.

Theorem 2. For $k \in \mathbb{N}_{\geq 3}, c \in[k-1]_{0}, i \in[c]_{0}$, and a weakly-c-balanced word $w \in \Sigma^{2 k-c}$, we have $\left|\operatorname{ScatFact}_{k-i}(w)\right| \geq k-c+1$, where equality holds if and only if $w \in\left\{\mathrm{a}^{k} \mathrm{~b}^{k-c}, \mathrm{a}^{k-c} \mathrm{~b}^{k}, \mathrm{~b}^{k} \mathrm{a}^{k-c}, \mathrm{~b}^{k-c} \mathrm{a}^{k}\right\}$. Moreover, if $w \in \Sigma_{w z b}^{2 k} \backslash\left\{\mathrm{a}^{k} \mathrm{~b}^{k}\right\}$, then $\left|\operatorname{ScatFact}_{k}(w)\right| \geq k+3$.

Remark 3. Theorem 2 answers immediately the question, whether a given set $S \subseteq \Sigma^{k}$, with $|S|<k+1$ or $|S|=k+2$, is a $k$-spectrum of a word $w \in \Sigma_{w z b}^{2 k}$ in the negative.

Theorem 2 shows that the smallest cardinality of the $k$-spectrum of a word $w$ is reached when the letters in $w$ are nicely ordered, both for weakly-0-balanced words as well as for weakly-c-balanced words with $c>0$. The largest cardinality is, not surprisingly, reached for words where the alternation of $a$ and $b$ letters is, in a sense, maximal, e.g., for $w=(\mathrm{ab})^{k}$. To this end, one can show a general result.

Theorem 4. For $w \in \Sigma^{*}$, the $k$-spectrum of $w$ is $\Sigma^{k}$ if and only if

$$
\{\mathrm{ab}, \mathrm{ba}\}^{k} \cap \operatorname{ScatFact}_{2 k}(w) \neq \emptyset .
$$

The previous theorem has an immediate consequence, which exactly characterises the weakly-0-balanced words of length $2 k$ for which the maximal cardinality of $\operatorname{ScatFact}_{k}(w)$ is reached.

Proposition 5. For $k \in \mathbb{N}_{\geq 3}$ and $w \in \Sigma_{w z b}^{2 k}$ we have $w \in\{\mathrm{ab}, \mathrm{ba}\}^{k}$ if and only if $\operatorname{ScatFact}_{k}(w)=\Sigma^{k}$.

To see why from $w \in\{\mathrm{ab}, \mathrm{ba}\}^{k}$ it follows that $\operatorname{ScatFact}_{k}(w)=\Sigma^{k}$, note that, by definition, a word $w \in\{\mathrm{ab}, \mathrm{ba}\}^{k}$ is just a concatenation of $k$ blocks from $\{\mathrm{ab}, \mathrm{ba}\}$. To construct the scattered factors of $w$, we can simply select from each block either the a or the $\mathrm{b}$. The resulting output is a word of length $k$, where in each position we could choose freely the letter. Consequently, we can produce all words in $\Sigma^{k}$ in this way. The other implication follows by induction.

Generalising Proposition 5 for weakly-c-balanced words requires a more sophisticated approach. A generalisation would be to consider $w \in\{\mathrm{ab}, \mathrm{ba}\}^{k-c} \mathrm{a}^{c}$. By Theorem 4 we have $\operatorname{ScatFact}_{k-c}(w)=\Sigma^{k-c}$. But the size of $\operatorname{ScatFact}_{k-i}(w)$ for $i \in[c]_{0}$ depends on the specific choice of $w$. To see why, consider the words $w_{1}=$ baabba and $w_{2}=(\mathbf{b a})^{3}$. Then by Proposition $5,\left|\operatorname{ScatFact}_{3}\left(w_{1}\right)\right|=8=$ $\left|\operatorname{ScatFact}_{3}\left(w_{2}\right)\right|$. However, when we append an a to the end of both $w_{1}$ and $w_{2}$, we see that in fact $\left|\operatorname{ScatFact}_{4}\left(w_{1} \mathbf{a}\right)\right|=11 \neq 12=\left|\operatorname{ScatFact}_{4}\left(w_{2} \mathbf{a}\right)\right|$. The main difference between weakly-0-balanced and weakly-c-balanced words for $c>0$, 
regarding the maximum cardinality of the scattered factors-sets, comes from the role played by the factors $\mathrm{a}^{2}$ and $\mathrm{b}^{2}$ occurring in $w$.

In the remaining part of this section we present a series of results for weakly$c$-balanced words. Intuitively, the words with many alternations between $\mathrm{a}$ and $\mathrm{b}$ have more distinct scattered factors. So, we will focus on such words mainly. Our first result is a direct consequence from Theorem 4 . The second result concerns words avoiding $\mathrm{a}^{2}$ and $\mathrm{b}^{2}$ gives a method to identify efficiently the $\ell$-spectra of words which are prefixes of $(a b)^{\omega}$, for all $\ell$. Finally, we are able to derive a way to efficiently enumerate (and count) the scattered factors of length $k$ of $(\mathrm{ab})^{k-c} \mathrm{a}^{c}$.

Corollary 6. For $k \in \mathbb{N}_{>3}, c \in[k]_{0}$, and $w \in \Sigma^{2 k-c}$ weakly-c-balanced, the cardinality of $\operatorname{ScatFact}_{k-c}(w)$ is exactly $2^{k-c}$ if and only if $\operatorname{ScatFact}_{2(k-c)}(w) \cap$ $\{\mathrm{ab}, \mathrm{ba}\}^{k-c} \neq \emptyset$.

As announced, we further focus our investigation on the words $w=(\mathrm{ab})^{k-c} \mathrm{a}^{c}$. By Theorem 4 we have $\left|\operatorname{ScatFact}_{i}(w)\right|=\Sigma^{i}$ for all $i \in[k-c]_{0}$. For all $i$ with $k-c<i \leq k$, a more sophisticated counting argument is needed. Intuitively, a scattered factor of length $i$ of $(\mathrm{ab})^{k-c} \mathrm{a}^{c}$ consists of a part that is a scattered factor (of arbitrary length) of (ab) ${ }^{k-c}$ followed by a (possibly empty) suffix of as. Thus, a full description of the $\ell$-spectra of words that occur as prefixes of $(\mathrm{ab})^{\omega}$, for all appropriate $\ell$, is useful. To this end, we introduce the notion of a deleting sequence: for a word $w$ and a scattered factor $u$ of $w$ the deleting sequence contains (in a strictly increasing order) w's positions that have to be deleted to obtain $u$.

Definition 7. For $w \in \Sigma^{*}, \sigma=\left(s_{1}, \ldots, s_{\ell}\right) \in[|w|]^{\ell}$, with $\ell \leq|w|$ and $s_{i}<s_{i+1}$ for all $i \in[\ell-1]$, is a deleting sequence. The scattered factor $u_{\sigma}$ associated to a deleting sequence $\sigma$ is $u_{\sigma}=u_{1} \ldots u_{\ell+1}$, where $u_{1}=w\left[1 . . s_{1}-1\right], u_{\ell+1}=$ $w\left[s_{\ell}+1 . .|w|\right]$, and $u_{i}=w\left[s_{i-1}+1 . . s_{i}-1\right]$ for $2 \leq i \leq \ell$. Two sequences $\sigma, \sigma^{\prime}$ with $u_{\sigma}=u_{\sigma^{\prime}}$ are called equivalent.

For the word $w=$ abbaa and $\sigma=(1,3,4)$ the associated scattered factor is $u_{\sigma}=$ ba. Since ba can also be generated by $(1,3,5),(1,2,4)$ and $(1,2,5)$, these sequences are equivalent.

In order to determine the $\ell$-spectrum of a word $w \in \Sigma^{n}$ for $\ell, n \in \mathbb{N}$, we can determine how many equivalence classes does the equivalence defined above have, for sequences of length $k=n-\ell$. The following three lemmas characterise the equivalence of deleting sequences.

Lemma 8. Let $w \in \Sigma^{n}$ be a prefix of $(\mathrm{ab})^{\omega}$. Let $\sigma=\left(s_{1}, \ldots, s_{k}\right)$ be a deleting sequence for $w$ such that there exists $j \geq 2$ with $s_{j-1}<s_{j}-1$ and $s_{j}+1=s_{j+1}$. Then $\sigma$ is equivalent $\sigma^{\prime}=\left(s_{1}, \ldots, s_{j-1}, s_{j}-1, s_{j+1}-1, s_{j+2}, \ldots s_{k}\right)$, i.e., $\sigma^{\prime}$ is the sequence $\sigma$ where both $s_{j}$ and $s_{j+1}$ were decreased by 1 .

Lemma 9. Let $w \in \Sigma^{n}$ be a prefix of $(\mathrm{ab})^{\omega}$. Let $\sigma=\left(s_{1}, \ldots, s_{k}\right)$ be a deleting sequence for $w$. Then there exists an integer $j \geq 0$ such that $\sigma$ is equivalent to the deleting sequence $\left(1,2, \ldots, j, s_{j+1}^{\prime}, \ldots, s_{k}^{\prime}\right)$, where $s_{j+1}^{\prime}>j+1$ and $s_{i}^{\prime}>s_{i-1}^{\prime}+1$, for all $j<i \leq k$. Moreover, $j \geq 1$ if and only if $\sigma$ contained two consecutive positions or $\sigma$ started with 1 . 
Lemma 10. Let $w \in \Sigma^{n}$ be a prefix of $(\mathrm{ab})^{\omega}$. Let $\sigma_{1}=\left(1,2, \ldots, j_{1}, s_{j_{1}+1}^{\prime}\right.$, $\left.\ldots, s_{k}^{\prime}\right)$, where $s_{j_{1}+1}^{\prime}>j_{1}+1$ and $s_{i}^{\prime}>s_{i-1}^{\prime}+1$, for all $j_{1}<i \leq k$, and $\sigma_{2}=\left(1,2, \ldots, j_{2}, s_{j_{2}+1}^{\prime \prime}, \ldots, s_{k}^{\prime \prime}\right)$, where $s_{j_{2}+1}^{\prime \prime}>j_{2}+1$ and $s_{i}^{\prime \prime}>s_{i-1}^{\prime \prime}+1$, for all $j_{2}<i \leq k$. If $\sigma_{1} \neq \sigma_{2}$ then $\sigma_{1}$ and $\sigma_{2}$ are not equivalent (i.e., $u_{\sigma_{1}} \neq u_{\sigma_{2}}$ ).

Lemmas 8,9 , and 10 show that the representatives of the equivalence classes w.r.t. the equivalence relation between deleting sequences, introduced in Definition 7 , are the sequences $\left(1,2, \ldots, j, s_{j+1}^{\prime}, \ldots, s_{k}^{\prime}\right)$, where $s_{j+1}^{\prime}>j+1$ and $s_{i}^{\prime}>s_{i-1}^{\prime}+1$, for all $j<i \leq k$. For a fixed $j \geq 1$, the number of such sequences is $\left(\begin{array}{c}(n-j-1)-(k-j)+1 \\ k-j\end{array}\right)=\left(\begin{array}{c}n-k \\ k-j\end{array}\right)$. For $j=0$, we have $\left(\begin{array}{c}(n-1)-k+1 \\ k\end{array}\right)=\left(\begin{array}{c}n-k \\ k\end{array}\right)$ nonequivalent sequences (note that none starts with 1 , as those were counted for $j=1$ already). In total, we have, for a word $w$ of length $n$, which is a prefix of $(\mathrm{ab})^{\omega}$, exactly $\sum_{j \in[k]_{0}}\left(\begin{array}{c}n-k \\ k-j\end{array}\right)$ nonequivalent deleting sequences of length $k$, so $\sum_{j \in[k]_{0}}\left(\begin{array}{c}n-k \\ k-j\end{array}\right)$ different scattered factors of length $n-k$. In the above formula, we assume that $\left(\begin{array}{l}a \\ b\end{array}\right)=0$ when $a<b$.

Moreover, the distinct scattered factors of length $\ell=n-k$ of $w$ can be obtained efficiently as follows. For $j$ from 0 to $\ell$, delete the first $j$ letters of $w$. For all choices of $\ell-j$ positions in $w[j+1 . . n]$, such that each two of these positions are not consecutive, delete the letters on the respective positions. The resulted word is a member of $\operatorname{ScatFact}_{\ell}(w)$, and we never obtain the same word twice by this procedure. The next theorem follows from the above.

Theorem 11. Let $w$ be a word of length $n$ which is a prefix of $(\mathrm{ab})^{\omega}$. Then $\left|\operatorname{ScatFact}_{\ell}(w)\right|=\sum_{j \in[n-\ell]_{0}}\left(\begin{array}{c}\ell \\ n-\ell-j\end{array}\right)$.

A straightforward consequence of the above theorem is that, if $\ell \leq n-\ell$ then $\mid$ ScatFact $_{\ell}(w) \mid=2^{\ell}$. With Theorem 11, we can now completely characterise the cardinality of the $\ell$-spectra of the weakly-c-balanced word $(\mathrm{ab})^{k-c} \mathrm{a}^{c}$ for $\ell \leq k$.

Theorem 12. Let $w=(\mathrm{ab})^{k-c} \mathrm{a}^{c}$ for $k \in \mathbb{N}, c \in[k]_{0}$. Then, for $i \leq k-c$ we have $\left|\operatorname{ScatFact}_{i}(w)\right|=2^{i}$. For $k \geq i>k-c$ we have $\left|\operatorname{ScatFact}_{i}(w)\right|=$ $1+2^{k-c}+\sum_{j \in[(i+c)-k-1]_{0}} \mid$ ScatFact $_{i-j-1}\left((\mathrm{ab})^{k-c-1} \mathrm{a}\right) \mid$.

As in the case of the scattered factors of prefixes of $(a b)^{\omega}$, we have a precise and efficient way to generate the scattered factors of $w=(\mathrm{ab})^{k-c} \mathrm{a}^{c}$. For scattered factors of length $i \leq k-c$ of $w$, we just generate all possible words of length $i$. For greater $i$, on top of $\mathrm{a}^{i}$, we generate separately the scattered factors of the form $u \mathrm{ba}^{j}$, for each $j \in[i-1]_{0}$. It is clear that, in such a word, $|u|=i-j-1$, and if $j \geq c$ then $u$ must be a scattered factor of $(\mathrm{ab})^{k-j-1} \mathrm{a}$, while if $j<c$ then $u$ must be a scattered factor of $(\mathrm{ab})^{k-c-1} \mathrm{a}$. If $j \geq(i+c)-k$ then, by Theorem $11, u$ can take all $2^{i-j-1}$ possible values. For smaller values of $j$, we need to generate $u$ of length $i-j-1$ as a scattered factor of $(\mathrm{ab})^{k-c-1} \mathrm{a}$, by the method described after Proposition 5.

Nevertheless, Theorems 11 and 12 are useful to see that in order to determine the cardinality of the sets of scattered factors of words consisting of alternating as and bs or, respectively, of $(a b)^{k-c} a^{c}$, it is not needed to generate these sets effectively. 


\section{Cardinalities of $k$-Spectra of Weakly-0-Balanced Words}

In the last section a characterisation for the smallest and the largest $k$-spectra of words of a given length are presented (Theorem 2 and Proposition 5). In this section the part in between will be investigated for weakly-0-balanced words (i.e. words of length $2 k$ with $k$ occurrences of each letter). As before, we shall assume that $k \in \mathbb{N}_{>3}$. In the particular case that $k=3$, we have already proven that the $k$-spectrum with minimal cardinality has 4 elements and that the maximal cardinality is 8 . Moreover as mentioned in Remark 3 a $k$-spectrum of cardinality 5 does not exist for weakly-0-balanced words of length $2 k$. The question remains if $k$-spectra of cardinalities 6 and 7 exist, and if so, for which words.

Before showing that a $k$-spectrum of cardinality $2^{k}-1$ for weakly-0-balanced words of length $2 k$ also exists for all $k \in \mathbb{N}_{>3}$, we prove that only scattered factors of the form $\mathrm{b}^{i+1} \mathrm{a}^{k-i-1}$ for $i \in[k-2]_{0}$ (up to renaming, reversal) can be "taken out" from the full set of possible scattered factors independently, without additionally requiring the removal of additional scattered factors as well. In particular, if a word of length $k$ of another form is absent from the set of scattered factors of $w$, then $\left|\operatorname{ScatFact}_{k}(w)\right|<2^{k}-1$ follows.

Lemma 13. If for $w \in \Sigma_{w z b}^{2 k}$ there exists $u \notin \operatorname{ScatFact}_{k}(w)$ with $u \notin\left\{\mathrm{b}^{i} \mathrm{a}^{k-i} \mid\right.$ $i \in[k-1]\} \cup\left\{\mathrm{a}^{i} \mathrm{~b}^{k-i} \mid i \in[k-1]\right\}$, then $\left|\operatorname{ScatFact}_{k}(w)\right|<2^{k}-1$.

Proposition 14. For $k \in \mathbb{N}_{\geq 3}$ and $w \in \Sigma_{w z b}^{2 k}$, the set $\operatorname{ScatFact}_{k}(w)$ has $2^{k}-1$ elements if and only if $w \in\left\{(\mathrm{ab})^{i} \mathrm{a}^{2} \mathrm{~b}^{2}(\mathrm{ab})^{k-i-2} \mid i \in[k-2]_{0}\right\}$ (up to renaming and reversal). In particular $\operatorname{ScatFact}_{k}(w)=\Sigma^{k} \backslash\left\{\mathrm{b}^{i+1} \mathrm{a}^{k-i-1}\right\}$ holds for $w=$ $(\mathrm{ab})^{i} \mathrm{a}^{2} \mathrm{~b}^{2}(\mathrm{ab})^{k-i-2}$ with $i \in[k-2]_{0}$.

By Proposition 14 we get that 7 is a possible cardinality of the set of scattered factors of length 3 of weakly-0-balanced words of length 6 and, moreover, that exactly the words $\mathrm{a}^{2} \mathrm{~b}^{2} \mathrm{ab}$ and $a \mathrm{ab}^{2} \mathrm{~b}^{2}$ (and symmetric words obtained by reversal and renaming) have seven different scattered factors. The following theorem demonstrates that there always exists a weakly-0-balanced word $w$ of length $2 k$ such that $\left|\operatorname{ScatFact}_{k}(w)\right|=2 k$. Thus, for the case $k=3$ also the question if six is a possible cardinality of $\operatorname{ScatFact}_{3}(w)$ can be answered positively.

Theorem 15. The $k$-spectrum of a word $w \in \Sigma_{w z b}^{2 k}$ has exactly $2 k$ elements if and only if $w \in\left\{\mathrm{a}^{k-1} \mathrm{bab}^{k-1}, \mathrm{a}^{k-1} \mathrm{~b}^{k} \mathrm{a}\right\}$ holds (up to renaming and reversal). Moreover, there does not exist a weakly-0-balanced word $w \in \Sigma_{w z b}^{2 k}$ with a $k$ spectrum of cardinality $2 k-i$ for $i \in[k-2]$.

By Proposition 14 and Theorem 15 the possible cardinalities of $\operatorname{ScatFact}_{3}(w)$ for weakly-0-balanced words $w$ of length 6 are completely characterized. Theorem 15 determines the first gap in the set of cardinalities of $\left|\operatorname{ScatFact}_{k}(w)\right|$ for $w \in \Sigma_{w z b}^{2 k}$ : there does not exist a word $w \in \Sigma_{w z b}^{2 k}$ with $\left|\operatorname{ScatFact}_{k}(w)\right|=k+i+1$ for $i \in[k-2]$ and $k \geq 3$, since all words that are not of the form $\mathrm{a}^{k} \mathrm{~b}^{k}$, $\mathrm{b}^{k} \mathrm{a}^{k}, \mathrm{a}^{k-1} \mathrm{bab}^{k-1}$, or $\mathrm{a}^{k-1} \mathrm{~b}^{k} \mathrm{a}$ have a scattered factor set of cardinality at least $2 k+1$. As the size of this first gap is linear in $k$, it is clear that the larger $k$ is, the more unlikely it is to find a $k$-spectrum of a small cardinality. 
In the following we will prove that the cardinalities $2 k+1$ up to $3 k-4$ are not reachable, i.e. $3 k-3$ is the thirst smallest cardinality after $k+1$ and $2 k$ (witnessed by, e.g. $\mathrm{a}^{k-2} \mathrm{~b}^{k} \mathrm{a}^{2}$ ).

Lemma 16. For $i \in\left[\left\lfloor\frac{k}{2}\right\rfloor\right]$ and $j \in[k-1]$

$$
\begin{aligned}
& \text { - }\left|\operatorname{ScatFact}_{k}\left(\mathrm{a}^{k-i} \mathrm{~b}^{k} \mathrm{a}^{i}\right)\right|=k(i+1)-i^{2}+1 \text { for } k \geq 4, \\
& -\left|\operatorname{ScatFact}_{k}\left(\mathrm{a}^{k-1} \mathrm{~b}^{2} \mathrm{ab}^{k-2}\right)\right|=3 k-2, \\
& -\left|\operatorname{ScatFact}_{k}\left(\mathrm{a}^{k-2} \mathrm{~b}^{j} \mathrm{ab}^{k-j} \mathrm{a}\right)\right|=k(2 j+2)-6 j+2 \text { for } k \geq 5, \text { and } \\
& -\left|\operatorname{ScatFact}_{k}\left(\mathrm{a}^{k-2} \mathrm{~b}^{j} \mathrm{a}^{2} \mathrm{~b}^{k-j}\right)\right|=k(2 j+1)-4 j+2 \text {. }
\end{aligned}
$$

Notice that for $i \in\left[\left\lfloor\frac{k}{2}\right\rfloor\right]$ the sequence $(k(2 i+1)-4 i+2)_{i}$ is increasing and its minimum is $3 k-2$ while for $i \in\left[\left\lfloor\frac{k}{2}\right\rfloor\right]$ the sequence $(k(2 i+2)-6 i+2)_{i}$ is increasing and its minimum is $4 k-4$. The following lemma only gives lower bounds for specific forms of words, since, on the one hand, it proves to be sufficient for the Theorem 18 which describes the second gap, and, on the other hand, the proofs show that the formulas describing the exact number of scattered factors of a specific form are getting more and more complicated. It has to be shown that also words starting with $i$ letters a, for $i \in[k-3]$, have a $k$-spectrum of greater (as lower is already excluded) cardinality. By Lemma 16 only words with another transition from a's to b's need to be considered, $\left(w=\mathrm{a}^{r_{1}} \mathrm{~b}^{s_{1}} w_{1} \mathrm{a}^{r_{1}} \mathrm{~b}^{s_{2}}\right)$. W.l.o.g. we can assume $s_{1}$ to be maximal, such that $w_{1}$ starts with an a, and similarly, by maximality of $r_{2}$, ends with a $\mathrm{b}$, thus only words of the form $\mathrm{a}^{r_{1}} \mathrm{~b}^{s_{1}} \ldots \mathrm{a}^{r_{n}} \mathrm{~b}^{s_{n}}$ have to be considered, and by Proposition 5, it is sufficient to investigate $n<k$.

Lemma 17. - $\left|\operatorname{ScatFact}_{k}\left(\mathrm{a}^{k-2} \mathrm{~b}^{i} \mathrm{ab}^{j} \mathrm{ab}^{k-i-j}\right)\right| \geq 3 k-3$ for $i, j \in[k-2]$, $i+j \leq k-1$,

- $\left|\operatorname{ScatFact}_{k}\left(\mathrm{a}^{k-2} \mathrm{~b}^{s_{1}} \mathrm{a}^{r_{1}} \mathrm{~b}^{s_{2}} \mathrm{a}^{r_{2}} \mathrm{~b}^{s_{3}}\right)\right| \geq 3 k-4$ for $s_{1}+s_{2}+s_{3}=k, r_{1}+r_{2}=$ $2, s_{1}>0, r_{1}, r_{2}, s_{2}, s_{3} \geq 0$

- $\left|\operatorname{ScatFact}_{k}\left(\mathrm{a}^{r_{1}} \mathrm{~b}^{s_{1}} \ldots \mathrm{a}^{r_{n}} \mathrm{~b}^{s_{n}}\right)\right| \geq 3 k-3$ for $r_{1} \leq k-3, \sum_{i \in[n]} r_{i}=\sum_{i \in[n]} s_{i}=$ $k$, and $r_{i}, s_{i} \geq 1$.

By Lemmas 16 and 17 we are able to prove the following theorem, which shows the second gap in the set of cardinalities of ScatFact ${ }_{k}$ for words in $\Sigma_{w z b}^{2 k}$.

Theorem 18. For $k \geq 5$ there does not exist a word $w \in \Sigma_{w z b}^{2 k}$ with $k$-spectrum of cardinality $2 k+i$ for $i \in[k-4]$. In other words, i.e. between $2 k+1$ and $3 k-4$ is a cardinality-gap.

Going further, we analyse the larger possible cardinalities of ScatFact ${ }_{k}$, trying to see what values are achievable (even if only asymptotically, in some cases).

Corollary 19. All square numbers, greater or equal to four, occur as the cardinality of the $k$-spectrum of a word $w \in \Sigma_{w z b}^{2 k}$; in particular $\left|\operatorname{ScatFact}_{k}\left(\mathrm{a}^{\frac{k}{2}} \mathrm{~b}^{k} \mathrm{a}^{\frac{k}{2}}\right)\right|=$ $\left(\frac{k}{2}+1\right)^{2}$ holds for $k$ even.

Inspired by the previous Corollary, we can show the following result concerning the asymptotic behaviour of the cardinality of ScatFact ${ }_{k}$ for words of length $2 k$. 
Proposition 20. Let $i>1$ be a fixed (constant) integer. Let $d=\left\lfloor\frac{k}{i}\right\rfloor$ and $r=k-d i$, and $d^{\prime}=\left\lfloor\frac{k}{i-1}\right\rfloor$ and $r^{\prime}=k-d^{\prime}(i-1)$. Then the following hold:

- the word $\mathrm{a}^{r} \mathrm{~b}^{r}\left(\mathrm{a}^{d} \mathrm{~b}^{d}\right)^{i}$ has $\Theta\left(k^{2 i-1}\right)$ scattered factors of length $n$;

- the word $\mathbf{a}^{r} \mathrm{~b}^{r^{\prime}}\left(\mathrm{a}^{d} \mathrm{~b}^{d^{\prime}}\right)^{i-1} \mathrm{a}^{d}$ has $\Theta\left(k^{2 i-2}\right)$ scattered factors of length $n$.

Remark 21. Let $i$ be an integer, and consider $k$ another integer divisible by $i$. Consider the word $w_{k}=\left(a^{\frac{k}{i}} b^{\frac{k}{i}}\right)^{i}$. The exact number of scattered factors of length $k$ of $w_{k}$ equals to the number $C\left(k, 2 i, \frac{k}{i}\right)$ of weak $2 i$-compositions of $k$, whose terms are bounded by $\frac{k}{i}$, i.e., the number of ways in which $k$ can be written as a sum $\sum_{j \in[2 i]} r_{j}$ where $r_{j} \in\left[\frac{k}{i}\right]_{0}$. From Proposition 20 we also get that this number is $\Theta\left(n^{2 k-1}\right)$, but we also have:

$$
C\left(k, 2 i, \frac{k}{i}\right)=\sum_{0 \leq j<M}(-1)^{j}\left(\begin{array}{c}
2 i \\
j
\end{array}\right)\left(\begin{array}{c}
k+2 i-j\left(\frac{k}{i}+1\right)-1 \\
2 i-1
\end{array}\right),
$$

for $M=\frac{i(k+2 i-1)}{k+i}$. It is known that there exists a constant $E>0$ such that

$$
C\left(k, 2 i, \frac{k}{i}\right) \leq E \cdot \sum_{0 \leq j<M}(-1)^{j}\left(\begin{array}{c}
2 i \\
j
\end{array}\right)\left(k+2 i-j\left(\frac{k}{i}+1\right)-1\right)^{2 i-1} .
$$

The coefficient of $k^{2 i-1}$ in the right hand side of this inequality has to be positive. Consequently $\sum_{0 \leq j<M}(-1)^{j}\left(\begin{array}{c}2 i \\ j\end{array}\right)(i-j)^{2 i-1}>0$. This seems to be an interesting combinatorial inequality in itself.

One can also show as in Proposition 20 that the number of scattered factors of length $k$ of $w_{k}$, which have, at their turn, $(a b)^{i}$ as a scattered factor, is $\Theta\left(k^{2 i-1}\right)$. This number also equals the number $C^{\prime}\left(k, 2 i, \frac{k}{i}\right)$ of $2 i$-compositions of $k$ whose terms are strictly positive integers upper bounded by $\frac{k}{i}$, i.e., the number of ways in which $k$ can be written as a sum $\sum_{j \in[2 i]} r_{j}$ where $r_{j} \in\left[\frac{k}{i}\right]$. Just as above, from this we get $\sum_{0 \leq j<i}(-1)^{j}\left(\begin{array}{c}2 i \\ j\end{array}\right)(i-j)^{2 i-1}>0$. Again, this inequality seems interesting to us.

We will end this analysis with the conjecture that, in contrast to the first gap, which always starts immediately after the first obtainable cardinality, the last gap ends earlier the larger $k$ is. More precisely, if $w=\mathrm{a}^{2} \mathrm{~b}^{2}(\mathrm{ab})^{k-3-i} \mathrm{ba}(\mathrm{ab})^{i}$ for $k \in \mathbb{N}_{\geq 4}, i \in[k-2]_{0}$ then $\left|\operatorname{ScatFact}_{k}(w)\right|=2^{k}-2-i$.

At the end of this section, we will briefly introduce $\theta$-palindromes in this specific setting. Let $\theta: \Sigma^{*} \rightarrow \Sigma^{*}$ be an antimorphic involution, i.e. $\theta(u v)=$ $\theta(v) \theta(u)$ and $\theta^{2}$ is the identity on $\Sigma^{*}$. By $\Sigma=\{\mathrm{a}, \mathrm{b}\}$ only the identity and renaming are such mappings. The fixed points of $\theta$ are called $\theta$-palindromes $\left(\mathrm{ab}^{3} \cdot \theta(\mathrm{b})^{3} \theta(\mathrm{a})\right)$ and exactly the words where $w^{R}=\bar{w}$ holds. They were studied in different fields well (see e.g., [5], [8]). A word $w \in \Sigma_{w z b}^{2 k}$ is a $\theta$-palindrome iff either $w \in\left\{\mathrm{a} w^{\prime} \mathrm{b}, \mathrm{b} w^{\prime} \mathrm{a}\right\}$ for some $\theta$-palindrome $w^{\prime} \in \Sigma_{w z b}^{2(k-1)}$ or additionally $w=\mathrm{a}^{\frac{k}{2}} \mathrm{~b}^{k} \mathrm{a}^{\frac{k}{2}}$ in the case that $k$ is even. Two cardinality results for $\theta$-palindromes 
are presented in Lemma 16 and Corollary 19. We believe that persuing the $k$ spectra of $\theta$-palindromes may lead to a deeper insight of which cardinalities can be reached, but due to space restrictions we will only mention one conjecture here, which may already show that cardinalities are somehow propagating for $\theta$-palindromes. Notice that this conjecture implies that indeed similar to the second gap here $4 k-4$ is always reached but that in contrast to the second gap, the third gap is not of the form $4 k-4-i$ for $i \in[k-4]$.

Conjecture 22. The $k$-spectrum of $w=\mathrm{ab}^{k-1} \mathrm{a}^{k-1} \mathrm{~b}$ has $4(k-1)$ elements and moreover if $w^{\prime}=w^{R}$ with a $k$-spectrum of cardinality $\ell \in \mathbb{N}_{\geq 12}$ then the scattered factor set of a $w \mathrm{~b}$ has cardinality $2 \frac{1}{4} \ell-5$.

\section{Reconstructing Weakly-0-Balanced Words from their $k$-Spectra}

In the final section we consider the slightly different problem of reconstructing a word from its scattered factors, or more specifically in this case, $k$-spectra. More generally, we are interested in how much information about a (weakly-0balanced) word $w$ is contained in its scattered factors, and more precisely, which scattered factors are not necessary or useful for reconstructing the word $w$, or distinguishing it from others. Since $w$ is a scattered factor of itself, it is trivial that the scattered factor of length $|w|$ is sufficient to uniquely reconstruct $w$. On the other hand, all words over $\{a, b\}{ }^{*}$ containing both letters will have the same 1 -spectrum. Thus we see that the length of the scattered factors of a word $w$ plays a role in how much information about $w$ they contain. This relationship is described more precisely by the following result of Dress and Erdös [3] along with the fact that (cf. e.g. Proposition 5) a word of length $2 k$ is not uniquely determined by its scattered factors of length $k$.

Proposition 23 (Dress and Erdös [3]). If $\operatorname{ScatFact}_{k+1}(w)=\operatorname{ScatFact}_{k+1}\left(w^{\prime}\right)$ holds for $w, w^{\prime} \in \Sigma^{\leq 2 k}$ then $w=w^{\prime}$ follows.

In the proof of Proposition 23, a pivotal role is played by scattered factors which contain many as and a few bs or vice-versa. The question arises as to whether this is due to the fact that these scattered factors contain inherently more information about the structure of the whole word than e.g., weakly-0balanced ones. In the general case, the answer is, sometimes at least, yes: we cannot distinguish between e.g. two words in $\{a\}^{*}$ by their weakly-0-balanced scattered factors, as the only such factor is $\varepsilon$. The same problem arises for all words which have a sufficiently uneven ratio of as to bs.

However, if in addition we consider only weakly-0-balanced words, then the situation changes. We conjecture that in fact, for these words $w$, the weakly-0balanced scattered factors are just as informative about the $w$ as the unbalanced ones. More formally, we believe the following adaptation of Proposition 23 holds:

Conjecture 24. Let $k \in \mathbb{N}$. Let $k^{\prime}=k+1$ for odd $k$, and $k^{\prime}=k+2$ for even $k$. Let $w, w^{\prime} \in \Sigma_{w z b}^{2 k}$ such that $\operatorname{ScatFact}_{k^{\prime}}(w) \cap \Sigma_{w z b}^{k^{\prime}}=\operatorname{ScatFact}_{k^{\prime}}\left(w^{\prime}\right) \cap \Sigma_{w z b}^{k^{\prime}}$. Then $w=w^{\prime}$. 
While we do not resolve the conjecture, we give an example of a subclass of words for which it holds true, namely when there are at most two blocks of bs (and therefore by symmetry if there are at most two blocks of as).

Proposition 25. Let $k \in \mathbb{N}$. If $k$ is odd, then each word $w \in \mathrm{a}^{*} \mathrm{~b}^{*} \mathrm{a}^{*} \mathrm{~b}^{*} \mathrm{a}^{*} \cap$ $\Sigma_{w z b}^{2 k}$ is uniquely determined by the set $\operatorname{ScatFact}_{k+1}(w) \cap \Sigma_{w z b}^{k+1}$. Similarly, if $k$ is even, then each word $w \in \mathrm{a}^{*} \mathrm{~b}^{*} \mathrm{a}^{*} \mathrm{~b}^{*} \mathrm{a}^{*} \cap \Sigma_{w z b}^{2 k}$ is uniquely determined by the set ScatFact $_{k+2}(w) \cap \Sigma_{w z b}^{k+2}$.

The difficulty in proving Conjecture 24 seems to arise from the fact that, for different pairs of words $w, w^{\prime} \in \Sigma_{w z b}$, the set of scattered factors which distinguish them, namely the symmetric difference of $\operatorname{ScatFact}_{k}(w) \cap \Sigma_{w z b}^{k}$ and ScatFact $_{k}\left(w^{\prime}\right) \cap \Sigma_{w z b}^{k}$ (for appropriate $k$ ), varies considerably, unlike with the proof(s) of Proposition 23, where the set of distinguishing scattered factors is always made up words of the same form, regardless of the choice of $w$ and $w^{\prime}$. As an example, consider the words $w=$ ababab, $w^{\prime}=$ bababa, and $w^{\prime \prime}=$ ababba. Then the symmetric difference of $\operatorname{ScatFact}_{4}(w) \cap \Sigma_{w z b}^{4}$ and $\operatorname{ScatFact}_{4}\left(w^{\prime}\right) \cap$ $\Sigma_{w z b}^{4}$ is $\{$ aabb, bbaa $\}$. On the other hand, considering $\operatorname{ScatFact}_{4}\left(w^{\prime}\right) \cap \Sigma_{w z b}^{4}$ and ScatFact $_{4}\left(w^{\prime \prime}\right) \cap \Sigma_{w z b}^{4}$, the symmetric difference is $\{$ baab $\}$.

\section{Conclusions}

We have considered properties of $k$-spectra of weakly-0-balanced words. In particular, in Section 3 we give several insights into the structure of the set of all $k$-spectra of weakly-0-balanced words of length $2 k$ by considering for which numbers $n$ there exists $w$ such that the $k$-spectrum of $w$ has cardinality $n$. In particular, we characterise the first two gaps in the possibilities for each $k$ which are regular (in the sense that the first and second gaps are always from $k+2$ to $2 k-1$ and $2 k+1$ to $3 k-4$ (inclusive). On the other hand, we see that the third gap is considerably less regular and thus resists a natural characterisation.

In Section 4, we consider the task of reconstructing weakly-0-balanced words from their $k$-spectra. We note that this is, in a sense, as hard as in the general case, however, we also conjecture that even if we consider only the scattered factors which are also weakly-0-balanced, then the situation remains the same, in the sense that it can be achieved for the same choices of $k$. Resolving this conjecture appears to require some new approach however since the techniques for the general case are not easily adapted.

As mentioned at the end of Section 3 some of the weakly-0-balanced words are $\theta$-palindromes. Since the $\theta$-palindromes of length $2 k$ are constructible from the ones of length $2(k-1)$ (except for each even $k$ exactly one $\theta$-palindrome) we surmised that the structure and properties propagate. Moreover we expected that the knowledge of the word's second half helps in finding the cardinalities of the $k$-spectra. Nevertheless we were only able to get results for $\theta$-palindromes in the same manner as for the other words, but we still believe that the structure of the $\theta$-palindromes can reveal more insights with further work. 


\section{References}

1. J. Berstel and J. Karhumäki. Combinatorics on words - A tutorial. BEATCS: Bulletin of the European Association for Theoretical Computer Science, 79, 2003.

2. Joel D. Day, Pamela Fleischmann, Florin Manea, and Dirk Nowotka. $k$-spectra of weakly-c-balanced words. https://arxiv.org/abs/1904.09125, 2019.

3. A. W.M. Dress and P. Erdös. Reconstructing words from subwords in linear time. Annals of Combinatorics, 8(4):457-462, 2004.

4. Cees H. Elzinga, Sven Rahmann, and Hui Wang. Algorithms for subsequence combinatorics. Theor. Comput. Sci., 409(3):394-404, 2008.

5. S. Z. Fazekas, F. Manea, R. Mercas, and K. Shikishima-Tsuji. The pseudopalindromic completion of regular languages. Information and Compution, 239:222-236, 2014.

6. Simon Halfon, Philippe Schnoebelen, and Georg Zetzsche. Decidability, complexity, and expressiveness of first-order logic over the subword ordering. In Proc. LICS 2017, pages 1-12, 2017.

7. Š. Holub and K. Saari. On highly palindromic words. Descrete Applied Mathematics, 157:953-959, 2009.

8. L. Kari and K. Mahalingam. Watson-crick palindromes in dna computing. Natural Computing, 9(2):297-316, 2010.

9. M. Lothaire. Combinatorics on Words. Cambridge University Press, 1997.

10. J. Manŭch. Characterization of a word by its subwords. In Developments in Language Theory, pages 210-219. World Scientific, 1999.

11. A. Mateescu, A. Salomaa, and S. Yu. Subword histories and parikh matrices. Journal of Computer and System Sciences, 68(1):1-21, 2004.

12. G. Rozenberg and A. Salomaa, editors. Handbook of Formal Languages (3 volumes). Springer, 1997.

13. A. Salomaa. Connections between subwords and certain matrix mappings. Theoretical Computer Science, 340(2):188-203, 2005.

14. Georg Zetzsche. The complexity of downward closure comparisons. In Proc. ICALP 2016, volume 55 of LIPIcs, pages 123:1-123:14, 2016. 